\title{
Efecto de la segregación de energías en la hipótesis de la curva ambiental de kuznets. Un estudio para el caso Ecuatoriano.
}

\section{Effect of energy segregation in the kuznets environmental curve hypothesis. A study for the ecuadorian Case.}

Gabriela Cristina Andrade Rojas

Diana Verónica Ramón Tacuri

Diego Fernando Roldán Monsalve

Juan Pablo Sarmiento Jara

Universidad de Cuenca, Ecuador

Autor para correspondencia: gaby12andrade@gmail.com; dveronica.ramont@ucuenca.edu.ec; roldandie@yahoo.com; juan.sarmiento@ucuenca.edu.ec

Fecha de recepción: 29 de enero del 2019 - Fecha de aceptación: 1 de abril del 2019

Resumen: El presente estudio examina el impacto de variables económicas relacionadas con la energía, sobre la degradación ambiental en Ecuador, bajo el enfoque de la Curva Medioambiental de Kuznets (EKC). Se utilizó información temporal correspondiente al periodo 1980-2014 y se estimó una ecuación de corto plazo y una de largo plazo utilizando la metodología propuesta por Pesaran y Shin (1998), basada en el modelo autorregresivo de rezagos distribuidos (ARDL). Se analizó el impacto del consumo de electricidad de fuentes renovables, del gas natural seco y del de petróleo en términos per cápita, en relación con las emisiones de $\mathrm{CO}_{2}$ por consumo de energía. Los resultados no verifican la hipótesis EKC, y se constató que el consumo de energía de fuentes renovables no es significativo para explicar las emisiones. Los pronósticos bajo la metodología ARIMA para el 2025 indican un aumento de las emisiones de $\mathrm{CO}_{2}$ por el consumo de energía en el Ecuador.

Palabras clave: curva medioambiental de kuznets; degradación ambiental; rezagos autorregresivos distribuidos

\begin{abstract}
This study examines the impact of energy-related economic variables on environmental degradation in Ecuador under the Kuznets Environmental Curve (EKC) approach. Information corresponding to the period 1980-2014 was used and a short term equation and a long term equation were estimated using the methodology proposed by Pesaran and Shin (1998), based on the autoregressive model of distributed lags (ARDL). The impact of electricity consumption from renewable sources, dry natural gas and oil consumption in per capita terms were analysed in relation to $\mathrm{CO}_{2}$ emissions from energy consumption. The results do not verify the EKC hypothesis, and it was found that energy consumption from renewable sources is not significant to explain emissions. Forecasts under the ARIMA methodology for 2025 indicate an increase in $\mathrm{CO}_{2}$ emissions from energy consumption in Ecuador.
\end{abstract}

Key Words: environmental kuznets curve; environmental degradation; autoregressive distributed lag model 


\section{Introducción}

La degradación medioambiental es un problema global que ha tomado fuerza durante las últimas décadas como consecuencia de un inadecuado crecimiento y desarrollo económico, motivando muchas investigaciones que buscan determinar los antecedentes y efectos de esta problemática.

Este escenario ambiental no presenta la misma intensidad en todos los países. Según Zambrano-Monserrate et al. (2018), las economías desarrolladas son las más afectadas por la contaminación debido a su alto grado de industrialización; sin embargo, las economías en desarrollo son más vulnerables a los efectos del cambio climático, el mismo que encuentra muchas de sus causas en las actividades humanas que generan gases de efecto invernadero (GEI), los que, de acuerdo con Ozturk y Acaravci (2010), están constituidos en un 60\% por dióxido de carbono.

Uno de los conceptos más usados para entender la relación entre la degradación ambiental y el desarrollo económico es la Curva Medioambiental de Kuznets (EKC por sus siglas en inglés). La hipótesis de esta curva sostiene que, en una primera etapa de desarrollo industrial, la calidad del medioambiente empeora hasta llegar a un pico, luego del cual, a un mayor desarrollo le corresponde una mejora en esa calidad.

Por otra parte, muchas investigaciones académicas se han desarrollado bajo la hipótesis EKC, debiendo determinar previamente cómo medir la calidad del medioambiente desde diferentes perspectivas incluyendo el bienestar humano (Streimikiene, 2015). Aunque son muchos los factores que afectan, numerosos estudios utilizan básicamente el dióxido de carbono $\left(\mathrm{CO}_{2}\right)$ como su variable proxy para medir la degradación medioambiental (Kılıç \& Balan, 2018; Zambrano-Monserrate, Silva-Zambrano, Davalos-Penafiel, Zambrano-Monserrate, \& Ruano, 2018; Rodríguez-Méndez, Pena-Boquete, \& Pardo-Fernández, 2016). Esta estrategia metodológica se aplica debido a su relevancia en los gases de efecto invernadero, además de que el $\mathrm{CO}_{2}$ está relacionado en buena medida con las formas de vida y producción de cada economía.

Como en todas las regiones, también en América Latina existe un vínculo entre su crecimiento económico y el deterioro ambiental resultante. En la década pasada, esta región ha presentado una bonanza económica vinculada al auge de las commodities. Este tipo de economías, basadas en la extracción de recursos naturales, repercuten significativamente en la degradación medioambiental (CEPAL, 2018).

A nivel nacional, en Ecuador también se evidencia un vínculo entre su crecimiento económico, las repercusiones ambientales y sus políticas energéticas y ambientales. Así, a partir del año 1972, con el boom petrolero nacional, la estructura económica del país y su dinámica se anclaron en el sector petrolero, siendo éste el recurso primordial en la matriz de energía primaria (Ministerio Coordinador de Sectores Estratégicos, 2013).

Según el Banco Mundial (2017), Ecuador ha experimentado un crecimiento promedio del PIB de 4,3\% durante el periodo del 2006 al 2014, impulsado por los altos precios del petróleo. Mientras que, las emisiones de GEI tuvieron un incremento duplicado del $8.77 \%$ desde 2006 al 
2012. Como referencia, en el año 2012, el sector Energía emitió el 44,92\% del $\mathrm{CO}_{2}$ por quema de combustible (Ministerio del Ambiente del Ecuador, 2017).

Según Ministerio Coordinador de Sectores Estratégicos (2013), el consumo de energía ha crecido durante el periodo de 1970 al 2012, específicamente de fuentes como el petróleo y del gas natural, mientras que, de fuentes tradicionales como la leña, han disminuido notablemente.

\section{Esfuerzos Iniciales para Mitigar el Problema Ambiental}

Motivados por razones económicas más que por ambientales, en el periodo 1964-1976, se desarrolló el primer Plan Maestro de Electrificación con el proyecto Central Paute entrando en operación parcial en 1983, disminuyendo la costosa y contaminante generación térmica desde el $64 \%$ al 32\%. La fase final funcionó en 1991. Muy posteriormente vendrían nuevos proyectos hidroeléctricos como la Central Paute Mazar y la Central Paute Sopladora cuyas construcciones se iniciaron en el año 2005 y en el 2010 respectivamente (Corporación Eléctrica del Ecuador CELEC, s.f.).

En Ecuador, en el Plan Nacional de Desarrollo 2007-2010 consideró la sustentabilidad del patrimonio natural a través del desarrollo de sistemas de energías renovables o alternativas, bajo el enfoque de sostenibilidad social (Secretaría Nacional de Planificación y Desarrollo, 2007).

En el Título II de la Constitución de la República del Ecuador del año 2008, el Capítulo VII se considera los Derechos de la Naturaleza, siendo uno de los primeros países de América Latina en incluirla en su marco normativo. Además, en el art. 395 de la Constitución de la República del Ecuador del 2008 se reconocen los principios ambientales, donde el Estado garantizará un desarrollo sustentable que mantenga la biodiversidad y la capacidad de regeneración natural de los ecosistemas, aplicando también políticas de gestión ambiental y fomentando una participación de la sociedad.

Una de las políticas del Objetivo 7 del Plan Nacional para el Buen Vivir del año 20132017 se enfocó en promover de manera eficiente y en mayor medida, una participación de energías renovables sostenibles, para prevenir la contaminación ambiental (Secretaría Nacional de Planificación y Desarrollo, 2013).

En el Plan Nacional de Desarrollo se promueve la gobernanza sostenible de los recursos naturales no renovables y renovables, e incentiva a la reducción de la contaminación, mitigación de los efectos del cambio climático, entre otros (Secretaría Nacional de Planificación y Desarrollo, 2017).

En la última década, Ecuador se ha interesado mucho en la generación de energía renovable que le permita reducir las emisiones de dióxido de carbono y aprovechar sus recursos naturales eficientemente, a través de un cambio de matriz energética que contempla proyectos referentes a generación hidráulica, eólica y solar. En 2014 la generación térmica fue el 49,13\%, 
la hidráulica de 45,57\%, la de biomasa el 1,59\%, la eólica el 0,32\% y la solar el 0,7\% (Constante, 2016).

Para el cambio de la matriz energética en el Ecuador, se han realizado obras emblemáticas con el fin de reemplazar el consumo de combustibles fósiles por un 51,78\% por la producción de energía renovable, disminuyendo la emisión de dióxido de carbono, como lo indica la Agencia de Regulación y Control de Electricidad, ARCONET (2016).

\section{Diferenciar energías de fuentes renovables y no renovables}

Entre los autores que en estudios recientes de EKC han considerado a la energía renovable y al consumo de energía son Bölük y Mert (2015) en un estudio en Turquía, mientras que Zambrano-Monserrate et al. (2018) Lo hacen en un estudio para el caso peruano. Ambos estudios verifican la hipótesis de EKC. Sin embargo, replicando otros estudios, ZambranoMonserrate et al. (2016) Para el caso ecuatoriano, no incluyen el consumo de energías renovables sino el consumo de energía en general verificando igualmente la hipótesis EKC.

Consideramos que no discriminar el consumo de energía renovable de la no renovable es un limitante para estudios medioambientales. La energía proveniente de las generadoras hidroeléctricas o eólicas son mucho más limpias que las producidas mediante derivados del petróleo. De hecho, como verificaremos más adelante, esta distinción de energías incluida en nuestro modelo es una de las causas para obtener resultados diferentes de la hipótesis EKC para el caso ecuatoriano.

Cabe señalar que, en 2017, la producción eléctrica nacional superó los 28.000 GWh según la ARCONEL, de los cuales un $72 \%$ tuvieron fuente hidráulica, un $26 \%$ fuente térmica y un $2 \%$ corresponden a otras fuentes como biomasa, eólica, solar, etc. En el período 1999-2017, la generación nacional de fuente hidráulica se incrementó un 179\%. Por otra parte, el consumo per cápita de electricidad en 2016 fue de $1.143 \mathrm{kWh} /$ habitante, frente a los $638 \mathrm{kWh} / \mathrm{habitante}$ de 1999, según el Instituto Nacional de Estadísticas y Censos del Ecuador. En el caso de los hogares, los esfuerzos del gobierno para estimular la sustitución de cocinas a gas por eléctricas han sido poco efectivos. Todo esto se refleja en un incremento notorio de las emisiones per cápita de dióxido de carbono en los cuatro últimos años de la serie estudiada, como se puede observar en el Gráfico 1, y que justifica preguntarnos si, con estos ajustes de modelo y escenario, ¿es posible pronosticar el cumplimiento de la hipótesis EKC para el caso ecuatoriano?

\section{Revisión De Literatura}

La EKC es una variante del estudio propuesto por Kuznets (1955) sobre la relación entre crecimiento y desigualdad en los Estados Unidos. La hipótesis original sostenía que, en una primera etapa de crecimiento, la desigualdad aumenta, pero de manera desacelerada hasta alcanzar un punto máximo desde el cual, a mayor crecimiento le corresponde una menor desigualdad en términos de Gini.

Grossman y Krueger (1991) sostienen que la relación entre el deterioro medioambiental y el crecimiento económico presentan el mismo comportamiento de U invertida. Estos autores, en 
su estudio inicial (1991) examinaron la relación entre la calidad del aire, medida a través del dióxido de azufre y el esmog, y el crecimiento económico. Sus resultados evidenciaron la relación de U invertida planteada por Kuznets. Posteriormente, estos mismos autores (1995) estudiaron la relacionaron de cuatro indicadores de impacto ambiental con el crecimiento económico encontrando el mismo comportamiento de U invertida, cumpliéndose la hipótesis de Kuznets.

Estudios más recientes han dedicado mayores esfuerzos en los recursos estadísticos, siendo uno de ellos la metodología de rezagos autorregresivos distribuidos (ARDL). Así, por ejemplo, Bölük y Mert (2015) en el caso de Turquía, examinan el potencial de las fuentes energéticas renovables para reducir el impacto de las emisiones de GEI durante el periodo de 1961 al 2010, recurriendo a la metodología ARDL. La hipótesis de la EKC se verifica entre las emisiones de GEI con el ingreso y la generación de electricidad de fuentes renovables.

Ahmad et al. (2017) Investigaron la existencia de la curva de Kuznets en Croacia para el periodo trimestral de 1992 al 2011, utilizando el enfoque ARDL y el modelo de vectores de corrección de error (VECM). Los resultados confirmaron Curva Medioambiental de Kuznets entre las emisiones de dióxido de carbono y el crecimiento económico.

Zambrano-Monserrate et al. (2016) Analizan en Ecuador el período 1971-2011 la relación entre PIB, emisión de $\mathrm{CO}_{2}$ y consumo de energía encontrando que las elasticidades de largo plazo revelan la existencia de un EKC para Ecuador, estableciendo que el país aún se encuentra en la zona de deterioro creciente de la curva. Estos resultados discrepan de los encontrados en el presente estudio.

Zambrano-Monserrate et al. (2018) analizaron en Perú durante el período 1980-2011 la relación entre el PIB, las emisiones de dióxido de carbono del consumo de energía, el total de consumo de electricidad renovable, el consumo del gas natural seco y el consumo total del petróleo, todas las variables en términos per cápita. Al igual que Narayan y Narayan (2010) aplicaron la metodología ARDL y el modelo VECM. En este caso, los resultados no siguieron la relación de forma de $\mathrm{U}$ invertida.

\section{Datos y modelo}

El presente estudio busca conocer el impacto que genera el uso de energías renovables y no renovables en la degradación ambiental.

Basado en este contexto, se busca determinar el impacto de variables económicas relacionadas con el uso de energías renovable y no renovables, sobre la degradación ambiental medida en función de las emisiones de dióxido de carbono del consumo de energía en el Ecuador durante el periodo de 1980 al 2014; periodo en el cual verificaremos si se cumple la hipótesis de EKC, además de determinar la fase en la que se encuentra el Ecuador. Adicionalmente emitiremos proyecciones hasta el año 2025 con respecto a su calidad medioambiental.

Las series temporales que fueron utilizadas son: las emisiones de dióxido de carbono del consumo de energía per cápita (toneladas métricas), el consumo total de electricidad renovable 
(miles de kilovatios hora), el consumo de gas natural seco (miles de pies cúbicos), consumo total de petróleo (barriles por año) y la población total, que no se incluye directamente en el modelo, fue obtenida para la transformación de las mencionadas variables, a términos per cápita. Estas series fueron tomadas de la Administración de Información Energética de Estados Unidos (EIA) (2018), el producto interno bruto per cápita (en dólares a precios constantes del 2010) y la densidad poblacional ${ }^{1}$ (personas por kilómetro cuadrado) del Banco Mundial (2018).

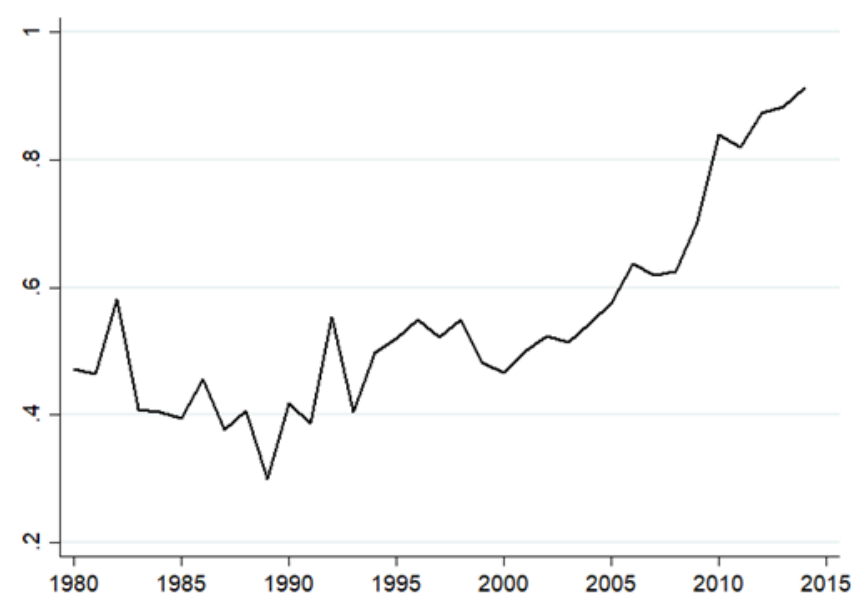

Gráfico 1: Indicador de Degradación Ambiental para Ecuador: Emisiones de Dióxido de Carbono del Consumo de Energía per cápita 1980-2014 (medido en logaritmos). Fuente: EIA (2018). Elaboración propia

El Gráfico 1 permite visualizar el comportamiento de las emisiones de dióxido de carbono del consumo de energía en el Ecuador. En la década de 1980 se puede apreciar una tendencia decreciente, sobre todo hasta 1989. A partir de este año, se muestra una tendencia creciente que responde al énfasis puesto sobre el sector energético. La tasa de crecimiento anual promedio de esta variable durante todo el periodo de estudio fue de 1,57\%. Cabe resaltar el significativo incremento en las emisiones observado fundamentalmente desde el año 2009.

\section{Especificación del modelo}

La siguiente especificación del modelo y desarrollo de la metodología se basa en el trabajo presentado por Zambrano-Monserrate et al. (2018).

Para esta investigación se considera el logaritmo natural (ln) de las variables en el modelo planteado. La ecuación (1) expresa la especificación de largo plazo (excluida la densidad

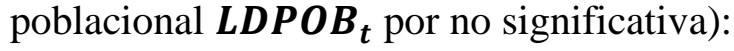

$$
\begin{aligned}
L_{C O 2 C E_{t}} & =\beta_{0}+\beta_{1} L P I B E C_{t}+\beta_{2} L P I B E C_{t}^{2}+\beta_{3} L C E R_{t}+\beta_{4} L G A S_{t}+\beta_{5} L C P E T_{t} \\
& +e_{t}
\end{aligned}
$$

Dónde: $L C O 2 C E$ es las emisiones de dióxido de carbono per cápita por consumo de energía; $L P I B E C_{t}$ es el PIB per cápita; $L C E R_{t}$ es el consumo de electricidad de fuentes

${ }^{1}$ Esta variable resulto no significativa por lo que no consta en el modelo final. 
renovables per cápita; $L C G A S_{t}$ es el consumo de gas natural seco per cápita; $L C P E T_{t}$ es el consumo de petróleo per cápita. En el modelo inicial se utilizó LPIBEC $_{t}^{2}$ (PIB per cápita al cuadrado), variable que busca capturar el comportamiento curvo (no lineal) de la EKC, sin embargo, esta resultó no significativa y con alta colinealidad por lo que, la prueba de dicha curvatura, para la hipótesis EKC, se resolvió por métodos alternos que se explican posteriormente en este mismo apartado. Así, la ecuación (1) se reduce a:

$$
L C O 2 C E_{t}=\beta_{0}+\beta_{1} L P I B E C_{t}+\beta_{2} L C E R_{t}+\beta_{3} L G A S_{t}+\beta_{4} L C P E T_{t}+e_{t}
$$

Los parámetros o elasticidades por estimar son $\boldsymbol{\beta}_{\mathbf{1}}, \boldsymbol{\beta}_{\mathbf{2}}, \boldsymbol{\beta}_{\mathbf{3}} \boldsymbol{y} \boldsymbol{\beta}_{\mathbf{4}}$, mientras que, $\boldsymbol{\beta}_{\mathbf{0}}$ es el término de intercepto y $\boldsymbol{e}_{\boldsymbol{t}}$ es el término de error con el supuesto de ser ruido blanco.

La especificación de este modelo (1b) se basa en la planteada por Narayan y Narayan (2010). Estos autores indican que, con este tipo de especificación, se evita caer en problemas de multicolinealidad. Además, para testear la existencia de la hipótesis de Kuznets, se comparan las elasticidades de los periodos de corto y largo plazo de la variable del PIB; de esta manera, si la elasticidad de largo plazo es mayor a la de corto plazo, no será posible asegurar la existencia de una relación de forma de U invertida, debido a que, en un largo plazo, el crecimiento del PIB indicará un incremento en las emisiones de dióxido de carbono (Zambrano-Monserrate, SilvaZambrano, Davalos-Penafiel, Zambrano-Monserrate, \& Ruano, 2018).

\section{Métodos}

\section{Cointegración y Rezagos Autorregresivos Distribuidos (ARDL)}

Coincidiendo con Ben Salha (2013), se deben distinguir los efectos de corto y largo plazo para lo que es adecuado el procedimiento ARDL propuesto inicialmente por Pesaran y Shin (1998) y desarrollado por Pesaran, Shin, y Smith (2001). Este enfoque para la cointegración tiene varias ventajas: en primer lugar, la relación a largo plazo entre dos variables se puede estimar incluso si las series tienen diferentes órdenes de integración, $I(0), I(1)$ o incluso integradas fraccionalmente (valores no enteros). En segundo lugar, ARDL no es sensible al tamaño de la muestra y puede ser aplicable incluso si es relativamente pequeña.

La presencia de estacionariedad en las series es testeada con las pruebas de raíz unitaria de Dickey-Fuller Aumentado (ADF) y Phillips-Perron.

La ecuación ARDL se especifica a continuación:

$$
\begin{aligned}
\Delta L C O 2 C E_{t}= & \alpha_{0}+\sum_{k=1}^{\pi_{1}} \alpha_{1 k} \Delta L C O 2 C E_{t-k}+\sum_{k=0}^{\pi_{2}} \alpha_{2 k} \Delta L P I B E C_{t-k}+\sum_{k=0}^{\pi_{3}} \alpha_{3 k} \Delta L C E R_{t-k} \\
& +\sum_{k=0}^{\pi_{4}} \alpha_{4 k} \Delta L C G A S_{t-k}+\sum_{k=0}^{\pi_{5}} \alpha_{5 k} \Delta L C P E T_{t-k}+\emptyset_{1} L C O 2 C E_{t-1} \\
& +\emptyset_{2} L P I B E C_{t-1}+\emptyset_{3} L C E R_{t-1}+\emptyset_{4} L C G A S_{t-1}+\emptyset_{5} L C P E T_{t-1} \\
& +\varepsilon_{t}
\end{aligned}
$$


Para determinar el orden óptimo de rezagos, se puede utilizar el criterio de información

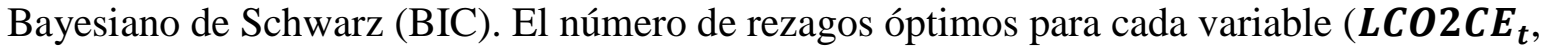

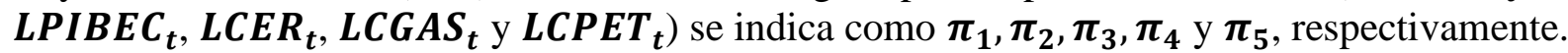
Pesaran, Shin y Smith (2001) utilizan un enfoque de cointegración basado en el modelo ARDL, denominado ARDL Bound Test. De esta manera, se plantea la prueba de cointegración en la que se deberá testear:

$\boldsymbol{H}_{0}: \emptyset_{1}=\emptyset_{2}=\emptyset_{3}=\emptyset_{4}=\emptyset_{5}=\mathbf{0}$ (no cointegra)

$H_{1}$ : al menos un $\varnothing$ es diferente de 0 (cointegra)

Para contrastar esta prueba se utiliza el estadístico F-Wald para compararlo con los valores críticos tabulados por Narayan (2005). En la tabla presentada por este autor, se muestran valores críticos $\boldsymbol{I}(\mathbf{0})$ e $\boldsymbol{I}(\mathbf{1})$ que representan los límites inferior y superior, obteniéndose tres regiones posibles con la siguiente interpretación del estadístico $\mathrm{F}$ :

$\mathrm{F}<$ límite inferior $I(0) \Longrightarrow$ No se rechaza $\mathrm{H}_{0}$, no hay cointegración

$\mathrm{F}>$ límite superior $I(1) \Longrightarrow$ Se rechaza $\mathrm{H}_{0}$, hay cointegración límite inferior $I(0)<\mathrm{F}<$ límite superior $I(1) \Longrightarrow$ no se obtienen una conclusión

La ecuación (3) de corto plazo se deriva de (1):

$$
\begin{aligned}
\Delta L C O 2 C E_{t} & =\alpha_{0}+\sum_{k=1}^{\pi_{1}} \alpha_{1 k} \Delta L C O 2 C E_{t-k}+\sum_{k=0}^{\pi_{2}} \alpha_{2 k} \Delta L P I B E C_{t-k}+\sum_{k=0}^{\pi_{3}} \alpha_{3 k} \Delta L C E R_{t-k} \\
& +\sum_{k=0}^{\pi_{4}} \alpha_{4 k} \Delta L C G A S_{t-k}+\sum_{k=0}^{\pi_{5}} \alpha_{5 k} \Delta L C P E T_{t-k}+\operatorname{\theta ECT}_{t-1}+u_{t}
\end{aligned}
$$

Donde:

$\mathbf{E C T}_{\boldsymbol{t}-\mathbf{1}}=$ es el término de corrección de error rezagado un período.

Para obtener $\mathbf{E C T}_{\boldsymbol{t}-\mathbf{1}}$ se debe calcular los residuos de (1), de tal forma que $\mathbf{E C T}_{\boldsymbol{t}}=\hat{\boldsymbol{e}}_{\boldsymbol{t}}$.

Dada la existencia de una relación de largo plazo, $\mathbf{E C T}_{\boldsymbol{t}-\mathbf{1}}$ indicará qué tan rápido las variables vuelven a sus niveles de equilibrio de largo plazo, a partir de variaciones de corto plazo. En este sentido, $\boldsymbol{\theta}$ deberá ser negativo y altamente significativo. Esta especificación de corto plazo es conocida también como el modelo de corrección de errores (ECM).

Pesaran y Shin (1998) demuestran que, cuando existe cointegración, los parámetros de MCO de corto plazo (que en este caso son los $\boldsymbol{\alpha}$ de la ecuación 3) son consistentes, inclusive con muestras pequeñas. Por otra parte, los estimadores de largo plazo (que son los $\boldsymbol{\beta}$ en la ecuación 1) son súper consistentes.

Zambrano-Monserrate et al. (2018) Apuntan que se puede testear también la estabilidad de los parámetros mediante la estimación recursiva de las pruebas de CUSUM y CUSUM cuadrado. Además, se podrá realizar las pruebas de diagnóstico referentes a la heterocedasticidad, la autocorrelación, la normalidad de los residuos y la especificación de Ramsey.

\section{Prueba de Causalidad de Granger}


En este caso, la prueba de causalidad de Granger es utilizada para conocer la dirección de la causalidad entre las variables de interés, teniendo en cuenta que el enfoque ARDL descrito previamente no la obtiene. A continuación, se muestra la estructura matricial del modelo de vector de corrección de error (VECM):

$$
(1-L) x_{t}=b+\sum_{i=1}^{\pi}(1-L) D_{i} x_{t-i}+\eta\left[E_{t-1}\right]+\epsilon_{t}
$$

Donde:

$\boldsymbol{x}_{\boldsymbol{t}} \quad$ Vector de variables del modelo

$\boldsymbol{b} \quad$ Vector de términos independientes

$\boldsymbol{D}_{\boldsymbol{i}} \quad$ Matriz de los coeficientes $\boldsymbol{d}^{\prime} \boldsymbol{s}$, asociados a los términos en diferencia con rezago $i$

$\boldsymbol{\eta} \quad$ Es el vector de coeficientes asociados al término $\mathbf{E C T}_{\mathbf{t}-\mathbf{1}}$

L Operador de rezagos

$(\mathbf{1}-\mathbf{L})=\boldsymbol{\Delta} \quad$ Es el operador de primera diferencia

Nuevamente, se puede determinar los rezagos óptimos mediante el criterio de información Bayesiano de Schwarz (BIC) o también, por el criterio de Akaike. Para testear la causalidad de Granger en el corto plazo, se estimarán los coeficientes $\boldsymbol{d}$, para posteriormente probar la siguiente hipótesis global:

$\boldsymbol{H}_{\mathbf{0}}: \boldsymbol{d}^{\prime} \boldsymbol{s}=\mathbf{0}$ (No existe causalidad de Granger)

Esta hipótesis nula podrá ser rechazada o no, dependiendo del valor de significación de las estadísticas F o del estadístico chi-cuadrado. En el caso de la causalidad de Granger en el largo plazo, se deberá estimar los coeficientes $\eta$, interpretándose de manera similar a la de la estimación del modelo de corrección de errores (ECM), tal que, los coeficientes de $\eta$ deberán ser negativos y altamente significativos para considerarse la existencia de una causalidad de Granger entre variables independientes y dependientes en el largo plazo (Zambrano-Monserrate et al., 2018).

\section{Pronóstico}

Con base en la metodología de un modelo autorregresivo, se estima el pronóstico de las variables independientes en el periodo de 2015-2025. Estas series permitirán proyectar las emisiones de dióxido de carbono por el consumo de energía para el Ecuador, mediante el pronóstico de modelos econométricos dinámicos realizado en STATA 15 (comando forecast solve), en la estimación del método ARDL.

\section{Resultados}

En primer lugar, se testeó la estacionariedad de las variables considerando que las series mantienen una tendencia determinística, donde los resultados de las pruebas de Dickey-Fuller Aumentada y Phillips-Perron, indicaron que todas las variables son integradas de orden 1, I(1), como se indica en la Tabla 1. 
Tabla 1: Resultados de la Prueba de Raíz Unitaria de Dickey-Fuller Aumentado y Phillip Perron

\begin{tabular}{|c|c|c|c|c|c|}
\hline & \multicolumn{2}{|l|}{ Nivel } & \multicolumn{3}{|c|}{ Primera Diferencia } \\
\hline Variable & Intercepto & $\begin{array}{l}\text { Intercepto y } \\
\text { tendencia }\end{array}$ & Intercepto & $\begin{array}{l}\text { Intercepto y } \\
\text { tendencia }\end{array}$ & $\begin{array}{l}\text { Orden de } \\
\text { Integración }\end{array}$ \\
\hline \multicolumn{6}{|l|}{$\begin{array}{l}\text { Dickey-Fuller } \\
\text { Aumentada }\end{array}$} \\
\hline$L C O 2 C E_{t}$ & $-0,383$ & $-2,315$ & $-9,527^{\star \star *}$ & $-10,253^{\star * *}$ & $I(1)$ \\
\hline$L P I B E C_{t}$ & 1,972 & $-0,351$ & $-4,710^{* * *}$ & $-5,965^{\star * *}$ & $I(1)$ \\
\hline$L C E R_{t}$ & $-3,030^{\star *}$ & $-2,487$ & $-4,029^{\star \star *}$ & $-4,464^{\star \star *}$ & $I(1)$ \\
\hline$L C G A S_{t}$ & $-0,894$ & $-2,855$ & $-7,226^{\star \star *}$ & $-7,244^{\star \star *}$ & $I(1)$ \\
\hline$L C P E T_{t}$ & 0,614 & $-2,11$ & $-5,848^{\star \star *}$ & $-6,472^{\star \star \star}$ & $I(1)$ \\
\hline \multicolumn{6}{|l|}{ Phillips-Perron } \\
\hline$L C O 2 C E_{t}$ & 0,442 & $-1,965$ & $-9,394^{\star * *}$ & $-11,648^{\star * *}$ & $I(1)$ \\
\hline$L P I B E C_{t}$ & 2,298 & $-0,167$ & $-4,769^{\star \star *}$ & $-6,006^{\star * *}$ & $I(1)$ \\
\hline$L C E R_{t}$ & $-3,219^{* *}$ & $-2,486$ & $-4,055^{\star \star *}$ & $-4,464^{* * *}$ & $I(1)$ \\
\hline$L C G A S_{t}$ & $-0,388$ & $-2,815$ & $-7,884^{\star * *}$ & $-8,138^{\star \star *}$ & $I(1)$ \\
\hline$L C P E T_{t}$ & 1,101 & $-1,928$ & $-5,879^{* * *}$ & $-6,961^{* * *}$ & $I(1)$ \\
\hline \multicolumn{6}{|l|}{ Notas: } \\
\hline \multicolumn{6}{|c|}{ Nivel de Significancia: $1 \%\left({ }^{* * *}\right), 5 \%\left({ }^{* *}\right)$ y $10 \%\left({ }^{*}\right)$. } \\
\hline \multicolumn{6}{|c|}{$\begin{array}{l}\text { El número de rezagos óptimo para Dickey-Fuller Aumentado se basa en el Criterio de } \\
\text { Información de Schwarz (SIC). } \\
\text { El número de rezagos óptimo para Phillips-Perron se basa en Newey- West. }\end{array}$} \\
\hline
\end{tabular}

\section{Elaboración propia}

Por lo tanto, se cumple el supuesto básico para la aplicación de la metodología ARDL, que se presenta a continuación.

\section{Equilibrio de Largo Plazo}

El enfoque del ARDL Bound Test permite encontrar una relación de largo plazo entre las emisiones de dióxido de carbono del consumo de energía y las variables planteadas. La Tabla 2 presenta los resultados de esta metodología en la cual los rezagos óptimos en el modelo son de $(1,4,2,4,2)$ según el criterio de Información bayesiano (BIC).

El estadístico F calculado (12,79) se contrastó con los valores críticos de Narayan (2005), siendo este mayor que el límite superior en todos sus niveles de significancia. Se consideraron los casos IV (con constante sin restricción y con tendencia restringida) y el caso V (con constante y tendencia, sin restricciones), debido al supuesto de tendencia determinística en las series (Pesaran, Shin, \& Smith, 2001). Este resultado confirmó la existencia de una relación de largo plazo en ambos casos.

Tabla 2: Resultados del ARDL Bound Test

\begin{tabular}{ll}
\hline $\begin{array}{ll}\text { Mod } \\
\text { elo }\end{array}$ & $\begin{array}{l}\text { CCO2CE}_{t} \\
=\boldsymbol{f}\left(\boldsymbol{L P I B E C}_{\boldsymbol{t}}, \boldsymbol{L C E R}_{t}, \boldsymbol{L C G} \boldsymbol{A} \boldsymbol{S}_{\boldsymbol{t}}\right.\end{array}$ \\
\hline & Rezagos ARDL: $(1,4,2,4,2)$ \\
\hline & Valores Críticos $(\mathrm{k}=4, \mathrm{n}=35)$
\end{tabular}




\begin{tabular}{llll}
\hline \multicolumn{3}{c}{ CASO V } \\
$\begin{array}{lll}\text { Límite } \\
\text { Inferior }\end{array}$ & $\begin{array}{l}\text { Límite } \\
\text { Superior }\end{array}$ & $\begin{array}{l}\text { CASO IV } \\
\text { Inferior }\end{array}$ & $\begin{array}{l}\text { Límite } \\
\text { Superior }\end{array}$ \\
\hline \multicolumn{1}{l}{$I(0)$} & $I(1)$ & $I(0)$ & $I(1)$ \\
\hline $1 \% \quad 5,604$ & 7,172 & 5,147 & 6,617 \\
\hline $5 \% \quad 4,036$ & 5,304 & 3,578 & 4,668 \\
\hline $10 \% \quad 3,374$ & 4,512 & 3,035 & 3,997 \\
\hline Estadístico $\mathrm{F}=12,799$ & & \\
\hline $\begin{array}{l}\text { Nota } \\
\text { S: }\end{array}$ & & \\
\hline $\begin{array}{l}\text { Valores críticos están basados en } \\
\text { Narayan (2005) }\end{array}$ \\
\hline $\begin{array}{l}\text { k= número de regresores no } \\
\text { determinísticos en la relación de } \\
\text { largo plazo }\end{array}$ \\
\hline
\end{tabular}

Elaboración propia.

En la Tabla 3 se presenta las estimaciones de largo plazo, en la cual, los coeficientes asociados a las variables representan las elasticidades de largo plazo. En este caso, el logaritmo del PIB, del consumo del gas y del consumo de petróleo en términos per cápita es estadísticamente significativos al menos al 5\% y 10\%. Con respecto a las variables logarítmicas del PIB y consumo de petróleo per cápita, presentan una relación positiva como se esperaba, de manera que, dado un aumento del $1 \%$ en el PIB y consumo petróleo per cápita, se generará un aumento del 2,43\% y 2,52\% en las emisiones de dióxido de carbono del consumo de energía, respectivamente. Mientras que, un aumento de $1 \%$ en el consumo de gas per cápita, disminuirá las emisiones de dióxido de carbono del consumo de energía en un $0,71 \%$; lo que en otros términos indica que un aumento de mil pies cúbicos en el consumo de gas per cápita generará una disminución de 0,71 toneladas métricas en las emisiones per cápita. En todo caso, este signo es contrario a lo esperado lo que podemos encontrar una posible explicación en que esta fuente sustituye a otras más contaminantes como carbón, leña o el mismo petróleo, lo cual requiere más evidencia.

La variable logarítmica del consumo per cápita de electricidad de fuentes renovables resultó no significativa en el modelo ( $p$-value $=0,589$ ). Sin embargo, se ha considerado importante incluirla en el modelo dado su relevancia en el estudio.

La estimación de corto plazo (Tabla 3) mostró que solo los coeficientes asociados al PIB y consumo de gas per cápita son significativos al $99 \%$ de confianza, siendo este último positivo. El término de corrección de error es negativo y significativo, lo que confirmó la hipótesis de cointegración. Este coeficiente implica que la velocidad de convergencia hacia el equilibrio de largo plazo es de $64,91 \%$.

Las elasticidades del PIB per cápita de largo plazo $(2,43 \%)$ y corto plazo $(-1,58 \%)$ indicaron que no se cumple la hipótesis de la curva medioambiental de Kuznets. Con este resultado, y bajo el enfoque planteado, no es posible determinar el punto de inflexión de la curva de medioambiental de Kuznets, y, por tanto, tampoco la fase en la que se encuentra el Ecuador. 
Se realizaron pruebas [véase Tabla 3] de diagnóstico para la validación del modelo, las cuales presentaron resultados favorables.

Tabla 3: Resultados de la Estimación ARDL de Corto y Largo Plazo

\begin{tabular}{|c|c|c|c|}
\hline \multirow[b]{2}{*}{ Variable dependiente: } & \multirow{2}{*}{$\frac{\text { Estimación de Largo plazo }}{\boldsymbol{L C O} \boldsymbol{C} \boldsymbol{E}_{\boldsymbol{t}}}$} & & \multirow{2}{*}{$\frac{\text { Estimación de corto plazo }}{\boldsymbol{D}\left(\boldsymbol{L C O} 2 \boldsymbol{C} \boldsymbol{E}_{\boldsymbol{t}}\right)}$} \\
\hline & & & \\
\hline \multirow[t]{2}{*}{$\operatorname{LPIBEC}_{t}$} & $2,430197 * * *$ & $D\left(L P I B E C_{t}\right)$ & $-1,583294 * * *$ \\
\hline & $(0,4084631)$ & & $(0,3784069)$ \\
\hline \multirow[t]{2}{*}{$L C E R_{t}$} & 0,1005342 & $D\left(L C E R_{t}\right)$ & $-0,0667695$ \\
\hline & $(0,1809412)$ & & $(0,0897694)$ \\
\hline \multirow[t]{2}{*}{$L C G A S_{t}$} & $-0,7097718 * *$ & $D\left(L C G A S_{t}\right)$ & $0,3890213 * * *$ \\
\hline & $(0,2344002)$ & & $(0,1141608)$ \\
\hline \multirow[t]{6}{*}{ LCPET $_{t}$} & $2,518285^{* *}$ & $D\left(\operatorname{LCPET}_{t}\right)$ & $-0,1734683$ \\
\hline & $(0,9918368)$ & & $(0,3669515)$ \\
\hline & & $\operatorname{ECT}(-1)$ & $-0,6491074 * * *$ \\
\hline & & & $(0,1327807)$ \\
\hline & & Constante & $36,27608 *$ \\
\hline & & & $(17,62773)$ \\
\hline R-cuadrado & 0,9571 & & \\
\hline R-cuadrado ajustado & 0,8927 & & \\
\hline \multicolumn{4}{|l|}{ Pruebas de Diagnóstico } \\
\hline & & Estadístico & P-value \\
\hline \multicolumn{2}{|c|}{ Autocorrelación (Breusch-Godfrey) } & 8,103 & 0,0879 \\
\hline \multicolumn{2}{|c|}{ Heterocedasticidad (White) } & 31 & 0,4154 \\
\hline \multicolumn{2}{|c|}{ Heterocedasticidad (ARCH) } & 2,245 & 0,1341 \\
\hline \multicolumn{2}{|c|}{ Especificación (Ramsey) } & 0,45 & 0,7242 \\
\hline \multicolumn{2}{|c|}{ Normalidad (Skewness/Kurtosis) } & 0,83 & 0,6617 \\
\hline
\end{tabular}

Notas:

Nivel de Significancia: $1 \%(* * *), 5 \%(* *)$ y $10 \%(*) .$. Entre paréntesis se presentan los errores estándar.

Los estadísticos corresponden al chi-cuadrado, a excepción del Test de Ramsey, que considera el F estadístico. Elaboración propia.
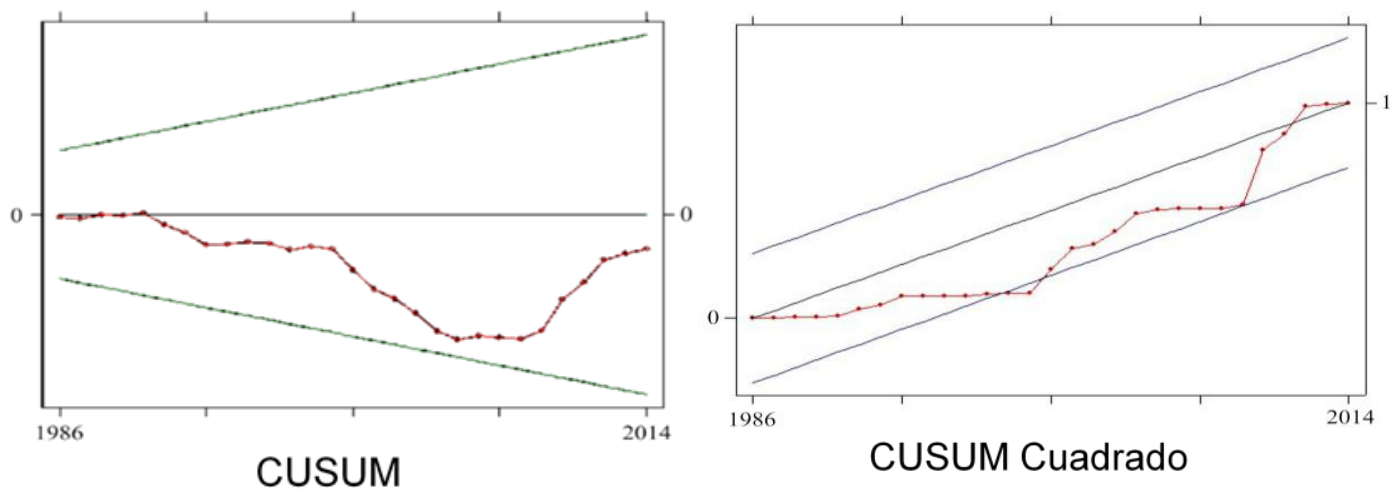

Gráfico 2: CUSUM y CUSUM Cuadrado. Fuente: Elaboración propia 
Las pruebas CUSUM y CUSUM cuadrado mostraron que los coeficientes estimados son estables para considerando el largo plazo, lo cual evidencia un modelo confiable (Gráfico 2).

\section{Relación de Causalidad}

Una vez que se determinó la presencia de integración entre $L C O 2 C E$ y $L P I B E C, L C E R$, $L C G A S$ y $L C P E T$ se testeó la dirección de la causalidad entre las variables a través del VECM con la prueba de causalidad de Granger. Se seleccionaron 4 rezagos óptimos según varios criterios, entre los que se presenta el criterio de información de Akaike (AIC), a través de la aplicación de un modelo VAR. Además, se determinaron 3 ecuaciones cointegrantes, según la prueba de cointegración de Johansen. Con estos resultados, se estimó el VECM para determinar la causalidad de largo plazo (Tabla 4). El coeficiente del término de corrección de error de la ecuación cointegrante 1 ( $\boldsymbol{L C O} \boldsymbol{C} \boldsymbol{C E}$ ) es negativo y significativo al 99\% de confianza, lo que confirma la relación de cointegración (Ver Tabla 5). Esta prueba de causalidad indica también que no existe evidencia de relación causal de largo plazo de las emisiones de $\mathrm{CO}_{2}$ hacia el PIB $(0,06994)$.

Tabla 4: Resultados de la Prueba de Causalidad de Largo Plazo Basado en el VECM.

\begin{tabular}{cc}
\hline Término de Corrección de Error $\left[\mathbf{E C T}_{\mathbf{t}-\mathbf{1}}\right]$ & $\boldsymbol{D}\left(\boldsymbol{L C O}_{\mathbf{C}} \boldsymbol{E}_{\boldsymbol{t}}\right)$ \\
\hline Eq. Coint. 1 & $-0,9777211^{* * *}$ \\
Eq. Coint. 2 & 0,5991674 \\
Eq. Coint. 3 & $-0,0823625$ \\
\hline
\end{tabular}

Nota: Nivel de Significancia: $1 \%(* * *), 5 \%(* *)$ y $10 \%(*)$

Elaboración propia.

En la Tabla 5 se presenta la prueba de causalidad de Granger la que indicó cuatro relaciones causales de corto plazo unidireccionales: de las emisiones de dióxido de carbono del consumo de energía, del consumo de electricidad de fuentes renovables, del consumo de gas natural y del consumo de petróleo, hacia el PIB real (todas en términos per cápita).

Tabla 5: Resultados de la Prueba de Causalidad de Granger en el VECM (corto plazo) (Chicuadrados).

\begin{tabular}{|c|c|c|c|c|c|}
\hline & $D\left(L C O 2 C E_{t}\right)$ & $D\left(\right.$ LPIBEC $\left._{t}\right)$ & $D\left(L C E R_{t}\right)$ & $D\left(L C G A S_{t}\right)$ & $\mathrm{D}_{\left(\mathrm{LCPET}_{t}\right)}$ \\
\hline$D\left(L C O 2 C E_{t}\right)$ & - & 4,84 & 2,96 & 2,98 & 3,84 \\
\hline$D\left(L_{P I B E C_{t}}\right)$ & $6,82^{*}$ & - & $10,09^{* *}$ & $8,86^{* *}$ & $8,82 * *$ \\
\hline$D\left(L C E R_{t}\right)$ & 2,29 & 0,82 & - & 0,58 & 0,83 \\
\hline$D\left(L_{C G A S_{t}}\right)$ & 1,42 & 1,17 & 0,79 & - & 3,81 \\
\hline$D\left(\right.$ LCPET $\left._{t}\right)$ & 2,56 & 1,24 & 2,57 & 1,16 & - \\
\hline
\end{tabular}

Nota: Se presenta el estadístico chi-cuadrado, con 3gl. Nivel de Significancia: 1\% (***), 5\% $(* *)$ y $10 \%(*)$.

Elaboración propia. 


\section{Pronóstico}

El pronóstico de las variables independientes $L P I B C E, L C E R, L C G A S, L C P E T$ para el periodo 2015-2025 se basa en la metodología del modelo autorregresivo, donde se asume un escenario de crecimiento promedio anual de $0,13 \% ; 0,71 \% ; 0,52 \%$ y $0,88 \%$ respectivamente. La Tabla 6 muestra los valores proyectados de las variables independientes.

Tabla 1: Tabla de proyecciones de las variables independientes

\begin{tabular}{lllllllll}
\hline $\boldsymbol{A N} \boldsymbol{N}$ & LPIBEC & LCER & LCGAS & LCPET & $\begin{array}{c}\text { PIBEC } \\
\text { (dólares) }\end{array}$ & $\begin{array}{c}\text { CER } \\
\text { (miles Kwh) }\end{array}$ & $\begin{array}{l}\text { GAS } \\
\text { (miles } \\
\text { pies } \\
\text { cúbicos) }\end{array}$ & $\begin{array}{c}\text { PET } \\
\text { (barriles } \\
\text { por año) }\end{array}$ \\
\hline $\mathbf{2 0 1 5}$ & 8,551 & 6,896 & 6,728 & 1,801 & 5171,754 & 988,519 & 835,097 & 6,058 \\
\hline $\mathbf{2 0 1 6}$ & 8,562 & 6,947 & 6,763 & 1,818 & 5229,554 & 1039,869 & 865,320 & 6,158 \\
\hline $\mathbf{2 0 1 7}$ & 8,573 & 6,997 & 6,799 & 1,834 & 5287,995 & 1093,886 & 896,637 & 6,260 \\
\hline $\mathbf{2 0 1 8}$ & 8,584 & 7,048 & 6,834 & 1,851 & 5347,095 & 1150,709 & 929,087 & 6,363 \\
\hline $\mathbf{2 0 1 9}$ & 8,595 & 7,099 & 6,870 & 1,867 & 5406,854 & 1210,484 & 962,712 & 6,468 \\
\hline $\mathbf{2 0 2 0}$ & 8,607 & 7,149 & 6,905 & 1,883 & 5467,282 & 1273,364 & 997,554 & 6,575 \\
\hline $\mathbf{2 0 2 1}$ & 8,618 & 7,200 & 6,941 & 1,900 & 5528,380 & 1339,510 & 1033,657 & 6,684 \\
\hline $\mathbf{2 0 2 2}$ & 8,629 & 7,251 & 6,976 & 1,916 & 5590,165 & 1409,092 & 1071,066 & 6,794 \\
\hline $\mathbf{2 0 2 3}$ & 8,640 & 7,301 & 7,012 & 1,932 & 5652,642 & 1482,289 & 1109,829 & 6,907 \\
\hline $\mathbf{2 0 2 4}$ & 8,651 & 7,352 & 7,048 & 1,949 & 5715,816 & 1559,289 & 1149,995 & 7,021 \\
\hline $\mathbf{2 0 2 5}$ & 8,662 & 7,403 & 7,083 & 1,965 & 5779,691 & 1640,288 & 1191,615 & 7,137 \\
\hline
\end{tabular}

Elaboración propia

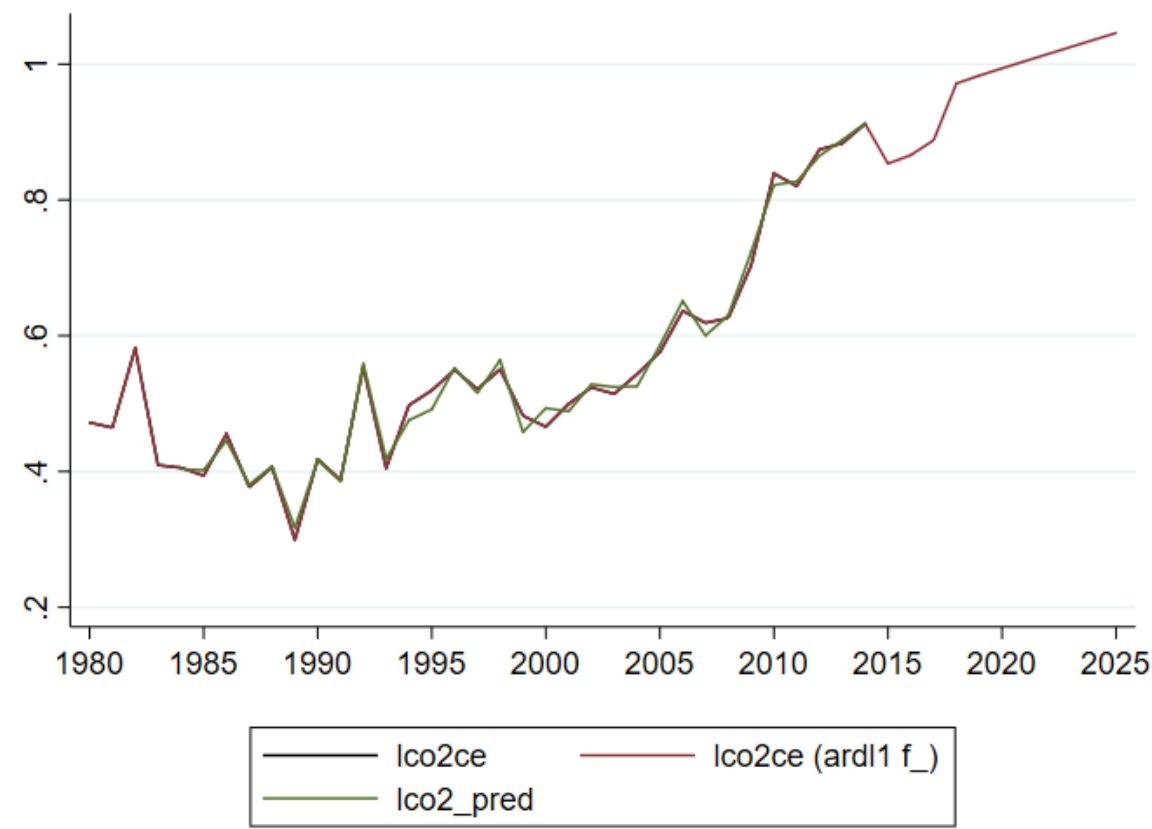

Gráfico 1: Pronóstico LCO2CE.

Fuente: Elaboración propia

Bajo este escenario, el Gráfico 3 muestra los resultados del pronóstico de las emisiones de dióxido de carbono del consumo de energía en el Ecuador durante el periodo 2015-2025 
(lco2ce ardl1 f_), los valores predichos del modelo ARDL en el periodo 1980-2014 (lco2_pred) y la serie original de las emisiones de $\mathrm{CO}_{2}$ del consumo de energía; todas estas presentadas en logaritmos y términos per cápita.

\section{Discusión}

En esta investigación se consideraron variables económicas relacionadas con las energías renovables y no renovables, además del PIB per cápita, sobre las emisiones de dióxido de carbono del consumo de energía en el Ecuador durante el periodo 1980-2014, bajo el enfoque de la Curva Medioambiental de Kuznets.

Se planteó el supuesto del cumplimiento de la curva EKC, de tal manera que, a medida que el país va creciendo económicamente, el factor medioambiental empeorará hasta que los ingresos lleguen a un punto de inflexión, a partir del cual, por la propia dinámica de la teoría de la EKC, la calidad ambiental mejorará.

Sin embargo, los resultados no permitieron verificar el cumplimiento de la hipótesis EKC, debido a que la elasticidad de largo plazo es mayor que la del corto plazo. Esto implica que las emisiones de dióxido de carbono del consumo de energía per cápita se incrementan con el tiempo a medida que existen mayores niveles de PIB per cápita. Además, el no cumplimiento de la hipótesis EKC impide predecir una futura disminución de la degradación ambiental frente a un mayor crecimiento económico, por lo tanto, en este caso el gobierno como ente legislativo, debería esforzarse en implementar instrumentos normativos y de regulación para que todos los sectores (público y privado) se orienten hacia un crecimiento económico con un manejo ambiental responsable ya que los datos analizados advierten que el deterioro ambiental no se corregirá por sí solo.

Por otra parte, estos resultados indican que el consumo per cápita de energías de fuentes no renovables como el gas natural seco y el petróleo muestra un impacto directo sobre las emisiones de $\mathrm{CO}_{2}$, mientras que el consumo de electricidad de fuentes renovables per cápita no presentó una incidencia significativa estadísticamente. Es posible que este escenario haya cambiado con la inauguración en 2016 de Coca Codo Sinclair, la mayor central hidroeléctrica del país, y que cubre el $30 \%$ del consumo nacional.

La relación atípica correspondiente al consumo de gas natural seco podría deberse a un efecto distorsionante de los subsidios relativos al gas que impiden una captura del comportamiento real de esta variable; así también, a un posible efecto sustitución entre el consumo de gas natural y leña como fuentes de energía, puesto que, esta última emite mayor contaminación que el consumo de gas. Se consideró también que, esta relación atípica podría estar afectada por una posible correlación negativa entre el consumo de gas y de petróleo, para lo cual se probó el modelo con la inclusión de una interacción entre estas dos variables, sin embargo, este coeficiente estimado tampoco fue significativo.

Los resultados también muestran que, siendo la elasticidad del consumo de petróleo mayor que la elasticidad de consumo de gas natural seco, en relación con las emisiones, reemplazar el consumo de petróleo por el de gas disminuiría las emisiones del $\mathrm{CO}_{2}$; sin embargo, 
una mejor alternativa ambiental y económica siempre será el uso de energías provenientes de centrales hidroeléctricas, campos eólicos y fotovoltaicos, conforme los objetivos del Plan Nacional de Desarrollo.

Nuestro estudio en Ecuador coincide parcialmente con los resultados de ZambranoMonserrate et al. (2018) en Perú, no verificándose la hipótesis EKC. Sin embargo, diferimos en que, en nuestro estudio, la variable del consumo de electricidad de fuentes renovables (LCER) no fue significativa y la del consumo de gas natural seco (LCGAS) mantiene una relación inversa con el deterioro ambiental en Ecuador.

Por otra parte, estos resultados también difieren de los de Zambrano-Monserrate (2016) para el caso ecuatoriano como consecuencia de desagregar en nuestro modelo las energías renovables de las no renovables y considerar adicionalmente el incremento per cápita de las emisiones de $\mathrm{CO}_{2}$ que traen como consecuencia el no cumplimiento de la EKC.

Los pronósticos realizados indican que, para el 2025 las emisiones de dióxido de carbono del consumo de energía aumentarán en el Ecuador posiblemente como consecuencia de una mayor tasa promedio anual de consumo de petróleo $(0,88 \%)$ en comparación con la tasa de crecimiento promedio anual del consumo de electricidad de fuentes renovables $(0,71 \%)$. Por lo tanto, los hacedores de políticas públicas en el Ecuador deberían enfocarse en la formulación de proyectos que faciliten el consumo de energías limpias lo cual es viable únicamente con el compromiso de la sociedad civil.

\section{Bibliografía}

Ahmad, N., Du, L., Lu, J., Wang, J., Li, H.-Z., \& Hashmi, M. Z. (2017). Modelling the CO2 emissions and economic growth in Croatia: Is there any environmental Kuznets curve? Energy, 123, 164-172.

Banco Mundial. (2017). Ecuador: Panorama General. Recuperado el 11 de abril de 2018, de Banco Mundial: http://www.bancomundial.org/es/country/ecuador/overview

Banco Mundial. (2018). Indicadores de Desarrollo Mundial. Recuperado el 15 de abril de 2018, de Banco de Datos del Banco Mundial: http://databank.bancomundial.org/data/reports.aspx? source=2\&series=NY.GDP.PCAP.C D\&country=\#

Ben Salha, O. (2013). Economic Globalization, Wages and Wage Inequality in Tunisia: An ARDL Bounds Testing Approach. Review of Middle East Economics and Finance, 9(3), 321-356. Doi: 10.1515/rmeef-2012-0037

Bölük, G., \& Mert, M. (2015). The renewable energy, growth and environmental Kuznets curve in Turkey: an ARDL approach. Renewable and Sustainable Energy Reviews, 52, 587-95.

CEPAL. (4 de marzo de 2018). América Latina y el Caribe adoptan su primer acuerdo regional vinculante para la protección de los derechos de acceso en asuntos ambientales. 
Recuperado el 25 de abril de 2018, de Comunicado de Prensa de la CEPAL: https://www.cepal.org/es/comunicados/america-latina-caribe-adopta-su-primer-acuerdoregional-vinculante-la-proteccion

Constante, J. (10 de septiembre de 2016). Importancia de la energía eléctrica en el cambio de la matriz energética en Ecuador. El Telégrafo. Recuperado el 27 de abril de 2018, de https://www.eltelegrafo.com.ec/noticias/408/1/importancia-de-la-energia-electrica-en-elcambio-de-la-matriz-energetica-en-ecuador

Constitución de la República del Ecuador. (2008). Constitución de la República del Ecuador. Constitución de la República del Ecuador. Quito, Ecuador.

Corporación Eléctrica del Ecuador CELEC. (s.f.). Cronología Paute Integral. Obtenido de https://www.celec.gob.ec/hidropaute/perfil-corporativo/cronologia.html

Grossman, G. M., \& Krueger, A. B. (1991). Environmental impacts of a North American free trade agreement. National Bureau of Economic Research.

Grossman, G. M., \& Krueger, A. B. (Mayo de 1995). Economic Growth and the Environment. The Quarterly Journal of Economics, 110(2), 353-377.

Kilıç, C., \& Balan, F. (2018). Is There an Environmental Kuznets Inverted-U Shaped Curve? PANOECONOMICUS, 65(1), 79-94. doi:https://doi.org/10.2298/PAN150215006K

Kuznets, S. (1955). Economic growth and income inequality. The American economic review, 45(1), 1-28.

Ministerio Coordinador de Sectores Estratégicos. (2013). Balance Energético Nacional 2013. Año base 2012. Quito: MCSE.

Ministerio del Ambiente del Ecuador. (2017). Tercera Comunicación Nacional del Ecuador sobre Cambio Climático. Quito, Ecuador.

Narayan, P. K. (2005). The saving and investment nexus for China: evidence from cointegration tests. Applied Economics, 37(17), 1979-1990.

Narayan, P. K., \& Narayan, S. (2010). Carbon dioxide emissions and economic growth: Panel data evidence from developing countries. Energy Policy, 38, 661-666. doi:10.1016/j.enpol.2009.09.005

Ozturk, I., \& Acaravci, A. (2010). CO2 emissions, energy consumption and economic growth in Turkey. Renewable and Sustainable Energy Reviews, 14(9), 3220-3225.

Pesaran, M. H., \& Shin, Y. (1998). An Autoregressive Distributed Lag Modelling Approach to Cointegration Analysis. Econometric Society Monographs, 31, 371-413. 
Pesaran, M. H., Shin, Y., \& Smith, R. J. (2001). Bounds testing approaches to the analysis of level relationships. Journal of Applied Cconometrics, 16(3), 289-326. doi:10.1002/jae.616

Rodríguez-Méndez, M., Pena-Boquete, Y., \& Pardo-Fernández, J. C. (2016). Revisiting Environmental Kuznets Curves through the Energy Price Lenses. Energy Policy, 95, 3241. doi:https://doi.org/10.1016/j.enpol.2016.04.038

Secretaría Nacional de Planificación y Desarrollo. (2007). Plan Nacional de Desarrollo 2007-2010. Planificación para la Revolución Ciudadana.

Secretaría Nacional de Planificación y Desarrollo. (2013). Plan Nacional para el Buen Vivir 20132017. Todo el Mundo Mejor. Ecuador.

Secretaría Nacional de Planificación y Desarrollo. (2017). Plan Nacional de Desarrollo 2017-2021. Toda una Vida. Ecuador.

Streimikiene, D. (2015). Environmental indicators for the assessment of quality of life. Intellectual Economics, 9, 67-79. doi:https://doi.org/10.1016/j.intele.2015.10.001

Zambrano-Monserrate, M. A., García-Albán, F. F., \& Henk-Vera, K. A. (2016). Bounds Testing Approach to Analyze the Existence of an Environmental Kuznets Curve in Ecuador. International Journal of Energy Economics and Policy, 6(2), 159-166.

Zambrano-Monserrate, M. A., Silva-Zambrano, C. A., Davalos-Penafiel, J. L., ZambranoMonserrate, A., \& Ruano, M. A. (2018). Testing environmental Kuznets curve hypothesis in Peru: The role of renewable electricity, petroleum and dry natural gas. Renewable and $\begin{array}{llll}\text { Sustainable Energy } & \text { Reviews, } & \text { 82(3), }\end{array}$ doi:https://doi.org/10.1016/j.rser.2017.11.005 